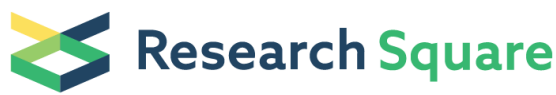 \\ Preprints are preliminary reports that have not undergone peer review. \\ They should not be considered conclusive, used to inform clinical practice, or referenced by the media as validated information.
}

\section{Novel Mutation of SIK1 Gene Causing a Mild Form of Pediatric Epilepsy in a Chinese Family}

\author{
Wangshu Xu \\ Beijing Tiantan Hospital \\ Wenqun Zhang \\ Chongqing Youyoubaobei women and chiledren's hospital \\ Lili Cui \\ Xuanwu Hospital \\ Lei Shi \\ Chinese People's Liberation Army Rocket Force Characteristic Medical Center \\ Bin Zhu \\ Beijing Tiantan Hospital \\ Tianjie Iv \\ China national clinical research center for neurological diseases \\ Wenping Ma ( $\nabla$ mawenping@bjmu.edu.cn) \\ Beijing Medical College: Capital Medical University https://orcid.org/0000-0002-1564-4584
}

\section{Research Article}

Keywords: SIK1 syndrome, Early infantile epileptic encephalopathy, genetic epilepsy, transcriptome, metabolome

Posted Date: November 22nd, 2021

DOI: https://doi.org/10.21203/rs.3.rs-1057581/v1

License: (c) (i) This work is licensed under a Creative Commons Attribution 4.0 International License. Read Full License 


\section{Abstract}

Objective: Early infantile epileptic encephalopathy (EIEE) is a group of disorders affecting children at early stages of infancy, which is characterized by frequent seizures, epileptiform activity on EEG, and developmental retardation or regression. Salt-inducible kinases (SIKs) syndrome is a newly described EIEE, caused by heterozygous mutations in the salt-inducible kinase SIK1, which can present as early myoclonic encephalopathy, Ohtahara syndrome, and infantile spasms.

Methods: In this study, we investigated a patient with early onset epilepsy. DNA sequencing of the whole coding region revealed a de nove/ heterozygous nucleotide substitution (c.880G>A) causing a missense mutation (p.A294T). This mutation was classified as variant of unknown significance (VUS) by American College of Medical Genetics and Genomics (ACMG). To further investigate the pathogenicity and pathogenesis of this mutation, we established a human neuroblastoma cell line (SH-SY5Y) stably-expressing wild type SIK1 and A294T mutant, and compared the transcriptome and metabolomics profiles.

Results: We presented a pediatric patient suffering from infantile onset epilepsy. Early EEG showed a boundary dysfunction of activity and MRI scan of the brain was normal. The patient responded well to single anti-epileptic drug treatment. Whole-exome sequencing found a missense mutation of SIK1 gene (c.880G>A chr21: 43420326 p. A294T). Dysregulated transcriptome and metabolome in cell models expressing WT and MUT SIK1 confirmed the pathogenicity of the mutation. Specifically, we found MEF2C target genes, certain epilepsy causing genes and metabolites are dysregulated by SIK1 mutation.

We found MEF2C target genes, certain epilepsy causing genes and metabolites are dysregulated by SIK1 mutation.

Significance: Our finding further expanded the disease spectrum and provided novel mechanistic insights of SIK1 syndrome.

\section{Introduction}

SIK1 syndrome is a newly described developmental epilepsy disorder (OMIM no. 616341) caused by de novo pathogenic sequence variations in the saltinducible kinase SIK1(Hansen et al., 2015). SIK1 thus is a novel genetic cause of EIEE. Up to date, there are few cases with SIK1 mutations in published literatures and the reported cases present as early myoclonic encephalopathy (EME), Ohtahara syndrome and infantile spasms(Hansen et al., 2015). The SIK1 syndrome spectrum and the mechanisms underneath remains poorly explored.

The SIK family includes SIK1-SIK3, which are characterized as serine/threonine kinases belonging to AMP-activated protein kinase (AMPK) family(F. Chen, Chen, Qin, \& Sun, 2019; H. Chen, Vanhoutte, \& Leung, 2019; Taub, Springate, \& Cutuli, 2010). Notably, SIK1 is highlyexpressed in the adrenal cortex, as well as in the adipose and neural tissues(F. Chen et al., 2019; Feldman et al., 2000; Horike et al., 2003). ACTH can induce phosphorylation of SIK1, which is dependent on Protein Kinase A (PKA) (Jaleel et al., 2006), resulting in translocationof activated SIK1 from the cytoplasm into the nucleus, where it furtherphosphorylates HDAC5(Al-Hakim et al., 2005). Phosphorylation of HDAC5 is required forMEF2C transcriptional activity to proceed(Bertorello \& Zhu, 2009). MEF2C is known as a transcription factor important in both dorsal and ventral neuronal developmental pathways(Du, Zheng, \& Pei, 2016).

SIKs and their substrates, such as CRTC and class Ila HDAC, are recently reported to be involved in gluconeogenesis(Sakamoto, Bultot, \& Goransson, 2018; Wein, Foretz, Fisher, Xavier, \& Kronenberg, 2018). SIKs inhibit gluconeogenesis, lipogenesis, steroidogenesis and the production of IL-10 via suppressing various gene expression. Protein and mRNA levels of SIK1 under fasting conditions were increased and SIK1 can inhibit gluconeogenesis in the hepatocytes. Besides, SIK2 inhibits transcriptional repressor ATF3 to upregulate GLUT4 expression, which leads to glucose uptake. Activity of SIKs kinase is regulated by energy deprivation and hormone presence (insulin, glucagon, and ACTH). In addition to its role in glucose metabolism, SIKs also seems to negatively regulate lipid metabolism(Sun, Jiang, Li, \& Guo, 2020). These metabolic roles of SIK1 in the pathogenesis of epilepsy have not been investigated.

Here we presented a pediatric patient suffering from infantile onset epilepsy. Early EEG showed a boundary dysfunction of activity and MRI scan of the brain was normal. The patient responded well to single anti-epileptic drug treatment. Whole-exome sequencing found a missense mutation of SIK1 gene (c.880G>A chr21: 43420326 p. A294T). The pathogenicity of the mutation was investigated by protein stability assay, transcriptome and metabolome profiling in cell models expressing WT and MUT SIK1. Indeed, mutant SIK1 leads to dysregulation of MEF2C target genes, certain epilepsy causing genes and metabolites. These findings strengthened our understanding of SIK1 syndrome.

\section{Materials And Methods}

\section{Patient}

The ethics committee of Beijing Tiantan Hospital of Capital Medical University approved the study. Written informed consent for participation in the study were obtained from patients' parents. Blood samples from patient and family members were collected for genetic studies, which were performed in accordance with the Declaration of Helsinki.

\section{Genetic Analyses}

Genomic DNA were isolated from peripheral blood samples. Disease-causing mutations were screened using whole genome sequencing (WES) (MyGenostics, Inc.). Candidate mutations were confirmed by Sanger sequencing using the following primers: seq F: 5'-CTGAGACAGCGGGTGCTGGAGG-3', seq R: 5'GGTCGGAAAGGGTCGGTGGAAA -3' .

\section{Construction of plasmids}


Full length human SIK1 cDNA template (NM173354) was purchased from YouBio (G112872), Shanghai China and subcloned to pLVX-Flag lentiviral expression plasmid and 7.1pCMV-3×Flag expression plasmid as wild type SIK1 (WT) using Seamless Cloning kit from Biomed (CL116), Beijing China. p.A294T mutant of SIK1 was generated by PCR method using WT as template. Mutations were confirmed by Sanger sequencing.

\section{Cells}

HEK 293T (Human Embryonic Kidney 293 cells transformed by expression of the large T antigen from SV40) (ATCC, CRL-11268) were cultured in Dulbecco's modified Eagle medium (DMEM). SH-SY5Y cells (human ATCC, CRL-2266) were cultured in F12K-DMEM.

\section{Stable cell lines establishment}

Lentivirus particles were produced by co-transfected HEK293T cells with pLVX-flag SIK1 WT and mutant plasmids (2.4 ug), packaging plasmids pCMV-VSV-G (800ng, AddGene 8454) and psPAX2 (800ng, AddGene 12260). The medium was changed to fresh DMEM containing $20 \%$ FBS at 24 hours post transfection and viral supernatant was collected at $48-72$ hours. Then, a total of $1 \times 10^{5} \mathrm{SH}-\mathrm{SY} 5 \mathrm{Y}$ cells were infected with viral supernatant supplemented with 8 ug/ml polybrene and incubated for 48 hours. Positive cells were screened by puromycin $(4 \mu \mathrm{g} / \mathrm{ml})$ and each monoclone was confirmed by western blot.

\section{Western blotting}

SH-SY5Y cells were harvested and lysed in RIPA buffer (Beyotime, P0013C), containing complete mini protease inhibitor cocktail (Roche, 04693124001). Proteins were separated by SDS-PAGE and transferred to polyvinylidene fluoride membranes (GE, PVDF 0.45UM, 10600023). Non-specific binding was blocked using $5 \%$ non-fat milk and the primary antibody was incubated at $4{ }^{\circ} \mathrm{C}$ overnight, the secondary antibody (anti-Flag, Sigma F3165) was incubated at room temperature for $1 \mathrm{~h}$. Signals were visualized by chemiluminescence (Millipore Corporation, Billerica, MA, USA).

\section{Cycloheximide Chase Assay}

This assay is adapted from previous report(Kao et al., 2015). Briefly, HEK293T cells were plated and transfected with various WT and mutant 7.1pCMV-3×Flag expression plasmids. 24 hours later, cycloheximide was added $(1 \mathrm{ug} / \mathrm{ml})$ and cells were collected at different time point for western blot detection.

\section{Transcriptome profiling}

Three monoclones of SH-SY5Y cells stably-expressing WT or Mutant SIK1 were subjected to whole genome RNA-sequencing. RNA was extracted using Trizol (Invitrogen, 15596-026, USA) according to the manufacturer's protocol. Total RNA was reverse transcribed using a RT-PCR Kit (Tiangen, KR103-03) according to the manufacturer's protocol. The sequencing reads were generated using the BGISEQ-500 platform following the manufacturer's recommendations. The paired-end clean reads were aligned to the reference human genome (UCSC version hg19) using TopHat v2.0.12. HTSeq v0.6.1 was used to count the read numbers mapped to each gene and the gene expression levels were calculated with RSEM version v1.2.31. FPKM of each gene was calculated based on the length of the gene and the read count mapped to that gene. We used MA plot, Volcano plot, Scatter plot and Heatmap plot to show the distributions of DEGs. The Holm's corrected P-value of 0.005 and log2 (fold change) of 1 were set as the threshold for significant differential expression. Then functional enrichment analysis was performed on GeneOntology (GO) and Kyoto Encyclopedia of Genes and Genomes (KEGG) pathway.

\section{Quantitative real-time PCR}

Total RNA was isolated with Trizol reagent (Invitrogen) and then reversed-transcribed with a Reverse Transcription System (Vazyme) following the manufacturer's protocol. The cDNA was amplified by an ABI 7500 Detection System using the AceQ qPCR SYBR Green Master Mix (Low ROX Premixed) (Vazyme) and the primers were listed below:

\begin{tabular}{ll} 
ARC F & AGCGGGACCTGTACCAGAC \\
\hline ARC R & GCAGGAAACGCTTGAGCTTG \\
\hline NR4A1 F & CCCTGAAGTTGTTCCCCTCAC \\
\hline NR4A1 R & GCCCTCAAGGTGTGGAGAAG \\
\hline GABRB3 F & GATAAAAGGCTCGCCTATTCTGG \\
\hline GABRB3 R & GATCATGCGGTTTTTCACTGTC \\
\hline SCN1AF & ATGTGGAAATAGCTCTGATGCAG \\
\hline SCN1A R & AGCCCAACTGAAGGTATCAAAG \\
\hline SCN2A F & TCTAAGCGTGTTTGCGCTAAT \\
\hline SCN2A R & ACCATTCCCATCCAATGAATTGT \\
\hline SCN9AF & AGAGGGGTACACCTGTGTGAA \\
\hline SCN9A R & CCCAGGAAAATCACTACGACAAA
\end{tabular}

Metabolome profiling 
Six monoclones of SH-SY5Y cells stably-expressing WT or Mutant SIK1 were subjected to metabolome profiling. LC-MS analysis were performed using a UHPLC system (1290, Agilent Technologies) with a UPLC HSS T3 column (1.8 $\mu \mathrm{m} 2.1 * 100 \mathrm{~mm}$, Waters) coupled to Q Exactive (Orbitrap MS, Thermo). The QE mass spectrometer was used for its ability to acquire MS/MS spectra on an information-dependent basis (IDA) during a LC/MS experiment. In this mode, acquisition software (Xcalibur 4.0.27, Thermo) continuously evaluates the full scan survey MS data as it collects and triggers the acquisition of MS/MS spectra depending on preselected criteria(Xiao, Zhou, \& Ressom, 2012).

MS raw data (.d) files were converted to the mzML format using ProteoWizard, and processed by R package XCMS (version 3.2). The preprocessed results generated a data matrix that consisted of the retention time (RT), mass-to-charge ratio $(\mathrm{m} / \mathrm{z})$ values, and peak intensity. Compound Discover (version 2.0, Thermo) and OSI-SMMS (version 1.0, Dalian ChemDataSolution Information Technology Co. Ltd.) was used for peak annotation after XCMS data processing with mzcloud database and in-house MS database(Wang et al., 2014, Xiao et al., 2012).

The peak number, sample name, and normalized peak area were fed to SIMCA14.1 software package (V14.1, MKS Data Analytics Solutions, Umea, Sweden) for principal component analysis (PCA) and orthogonal projections to latent structures-discriminate analysis (OPLS-DA). PCA showed the distribution of original data. In order to obtain a higher level of group separation and a better understanding of variables responsible for classification, supervised OPLS-DA were applied. 7-fold cross-validation was used to estimate the robustness and the predictive ability of our mode(Wiklund et al., 2008).

Based on OPLS-DA model, a loading plot was constructed, which showed the contribution of variables to the differences between two groups. It also showed the significant variables which were situated far from the origin, but the loading plot is complex because of many variables. To refine this analysis, the first principal component of variable importance in the projection (VIP) was obtained. In addition, commercial databases including KEGG http://www.genome.jp/kegg/ and MetaboAnalyst http://www.metaboanalyst.ca/ were utilized to search for the pathways of metabolites.

\section{Statistical analysis}

Samples were compared using two-tailed, unpaired Student's $t$-test with GraphPad Prism 7.00. Error bars were represented by SEM. ${ }^{\star} P<0.05,{ }^{\star \star} P<0.01,{ }^{\star \star \star} P<$ 0.001 .

\section{Results}

\section{Case description}

The patient is a 2+ year-old boy with normal spontaneous vaginal delivery and development. He presented the first seizure at 1 year and 3 months without obvious triggering factors. Since then, he experienced 9 more similar seizures in the following 2 months before receiving anti-epileptic treatment. The seizure patterns were all presented as generalized tonic-clonic seizures (GTCS) with 2 of them accompanying with fever of $38^{\circ} \mathrm{C}$.

All the seizures last for about 2-3 min and could remit spontaneously. No obvious abnormality was found on neurological examination and brain magnetic resonance imaging (MRI). One week later after the last seizuring, electroencephalogram monitoring showed a moderate increase of background 3-4Hz $\mathrm{d}$ and $\mathrm{q}$ mixed activity during awake and occasional spike-and-slow waves in bilateral prefrontal regions during sleep. Blood electrolyte, cerebrospinal fluid routine, biochemistry and pathological tests were all normal. The family history of seizure is negative. The patient was diagnosed of epilepsy and treated with sodium valproate. This single anti-epileptic treatment lasted until today (1 year and 3 months) and during follow-up his EEG return to and remain normal.

\section{Genetic findings}

A novel heterozygous SIK1 mutation (c.880G>A) was detected only in the proband by using whole-exome sequencing, and Sanger sequencing was used to validate it (Fig. 1A). Thus, this is a de novo mutation. This mutation (c.880G>A chr21: 43420326 p. A294T) is identified as "rs776214001" in dbSNP and occurs at a frequency of 0.000042 in the global population, of 0.00000 in Europe, American and African populations and of 0.00020 in Asian population in the ExAC database (https://www.ncbi.nlm.nih.gov/snp/rs776214001?vertical_tab=true\#frequency_tab) and has not been reported in previous study. Multiple sequence alignment was performed using Mega 7.0, and residue A294 is only highly conserved in primates (Fig. 1B), which may explain that three bioinformatics programs show that the novel mutation might be "Tolerated" (Table 1). The pathogenicity of this variant is classified as variant of unknown significance (VUS), following the principle of standards and guidelines recommended by ACMG(Li et al., 2017). Despite of this, this variant was still proposed as the causative mutation for the clinical phenotype due to its extremely low frequency in general populations and lacking of other de novo compelling disease-causing candidates, which is very similar to the first reported missense mutation cases(Hansen et al., 2015). To support this, we performed Cycloheximide Chase Assay to test the mutant protein stability. We showed that mutant protein was more resistant to degradation compared to wild type protein (Fig. 1C), similar to previous reported frameshifted mutants but not the missense mutants(Proschel et al., 2017).

\section{SH-SY5Y cells expressing mutant SIK1 showed significantly skewed transcriptome}

To gain more insights of the pathogenicity and pathogenesis of patient's mutation, we infected human neuroblastoma cell line (SH-SY5Y) with a Flag-epitopetagged wild-type or mutant SIK1 using a lentiviral vector. Next, we established 3 independent monoclonal SH-SY5Y cell lines stably-expressing wild type SIK1 and 3 independent monoclonal SH-SY5Y cell lines stably-expressing p.A294T mutant SIK1. Each cell line achieved robust expression as detected by western blot (Fig. 2A). All 6 cell lines were subjected to bulk RNA-sequencing and analyzed and compared as WT group and MUT group. Pearson correlation between samples showed that within each group samples were closely related (R2>92\%), while between groups samples were not significantly related (R2<80\%), indicating transcriptomes within groups are similar but transcriptomes between groups are significantly different (Fig. 2B). The significantly skewed transcriptome of cells expressing p.A294T mutant SIK1 were further supported by the large number of significantly differentially expressed genes (DEGs) as shown in Fig. 2C, with 3178 genes upregulated and 4505 genes downregulated. We next analyzed these DEGs with various enrichment methods. It is 
noteworthy that using DisGeNET enrichment method we found a cluster of dysregulated genes termed "INFANTILE_SEVERE_MYOCLONIC_EPILEPSY" (Fig. 2D). Pathogenic variants in SCN2A can causea spectrum of neurodevelopmental disorders, such as developmental and epileptic encephalopathies, benign familial neonatal-infantile seizures, episodic ataxia, and autism spectrum disorder and intellectual disability with and without seizures(Reynolds, King, \& Gorman, 2020). SCN1A mutations were reported to be responsible for genetic epilepsy with febrile seizures plus (GEFS(+)) in multiplex families and accounts for $70-80 \%$ of Dravet syndrome (DS)(Marini et al., 2007). The gamma-aminobutyric acid type A receptor $\beta 3$ gene (GABRB3) encodes the $\beta 3$-subunit of the gamma-aminobutyric acid type A (GABAA) receptor, andmediates inhibitory signaling within the central nervous system. Recently, GABRB3 mutations have been identified in a few patients with infantile spasms and Lennox-Gastaut syndrome(Papandreou et al., 2016). In addition, other clusters relating to SIK1 syndrome disease spectrum were also enriched by this method including "AUTONOMIC_NERVOUS_SYSTEM_DISORDERS" and "AUTISTIC_BEHAVIOR" (Fig. 2E and 2F). Disease Ontology (DO) enrichment method also showed another cluster of dysregulated genes termed "TEMPORAL_LOBE_EPILEPSY" (Fig. 2G). Further we confirmed some of these dysregulated genes with RT-qPCR which are well known to caused epilepsy (Fig. $2 \mathrm{H})$. Pathogenic mutations of SIK1 were associated with decreased expression of ARC and NR4A1, both are synaptic activity response element genes.(Proschel et al., 2017). Consistently, cells expressing p.A294T mutant SIK1 showed decreased expression of ARC and NR4A1 (Fig. 2I). Taken together, these data further confirmed the pathogenicity of the SIK1 mutation of our patient and indicated the mechanism of the epilepsy caused by this mutation might be similar with reported cases at least to certain extent.

\section{SH-SY5Y cells expressing mutant SIK1 showed significantly skewed metabolome}

SIKs belong to AMP-activated protein kinase (AMPK) family, and functions mainly involve in regulating energy response-related physiological processes, such as gluconeogenesis and lipid metabolism(Sakamoto et al., 2018). To investigate the metabolic consequences of SIK1 mutation in our patient, 6 independent monoclonal SH-SY5Y cell lines stably-expressing wild type SIK1 and 6 independent monoclonal SH-SY5Y cell lines stably-expressing p.A294T mutant SIK1. Cells were subjected to nontarget metabolomics profiling and analyzed and compared as WT group and MUT group.

Principal component analysis (PCA) showed a significantly different metabolic pattern between WT group and MUT group (Fig. 3A). Orthogonal projections to latent structures-discriminant analysis (OPLS-DA) (Fig. 3B), which a supervised multivariate statistical analysis with better discriminative power, and Permutation test of OPLS-DA model (Fig. 3C) further confirmed this. Synchronous permutation test can eliminate the overfitting effect of the model.

We then analyzed the differentially expressed metabolites between groups and we found there were large number of metabolites upregulated or downregulated in MUT group compared to WT group (Fig. 3D). The top upregulated (Table 2) and downregulated (Table3) metabolites were listed. Notably, Chondroitin 6'-sulfate was significantly increased and Adenosine was significantly decreased in mutant SIK1-expressing cells. The general quantification of differentially expressed metabolites between groups were shown in the radar chart (Fig. 3E). Further pathway enrichment analysis showed thiamine and purine metabolism pathways were deeply involved (Fig. 3F).

\section{Discussion}

To our best knowledge, only 6 cases of SIK1 syndrome have been reported before our report(Hansen et al., 2015), thus our understanding of this disease is rather limited. In this study we presented the seventh case worldwide of SIK1 syndrome. The clinical and mechanistical investigations we made in our patient added novel knowledge on SIK1 syndrome.

In the first and only case series report, the authors demonstrated that 3 of 6 cases presented as infantile spasm syndrome, 2 of 6 cases presented as Early Myoclonic Epilepsy (EME) starting within the first hour of life with suppression-burst pattern on EEG and 1 of 6 cases presented as Ohtahara syndrome. These are well known hard-to-treat, very severe forms of epilepsy. Interestingly, our patient, bearing a novel missense mutation of SIK1, showed a mild form of epilepsy and good response to antiepileptic drug treatment. Consistently, EEG test of our patient only showed boundary changes at early disease course and soon returned to normal after antiepileptic drug treatment, while the EEG test of the index patient in the previous report showed burst suppression pattern with myoclonic activity and did not improve after a long period of treatment. The MRI scan of SIK1 syndrome patients varied from normal to obvious morphology changes. Our patient showed normal morphology during the whole course. In addition, 3 previously reported subjects presenting with infantile spasms subsequently developed intractable epilepsy and an autism plus developmental disorder with absent speech, impaired socialization, and repetitive behaviors. Our patient is 2 years old now and show no sign of autism nor any other developmental disorders. Nevertheless, long term follow-up is needed to fully characterize the clinical phenotype of our patient.

On the aspects of mechanisms, it is reported that all truncated mutant SIK1 showed increased protein stability, abnormal cellular localization and increased activation activity on Histone deacetylase 5 (HDAC5) while all missense mutant SIK1 only showed increased activation activity on HDAC5. In contrast to this, our mutant showed resistance to degradation as previously reported truncated mutants (Fig. 1C). The SIK1 protein is integrated in a complex functional network that relays signaling through protein kinase A to various targets. HDAC5 is one of the best researched targets for SIK1 action(Wein et al., 2018).

Epilepsy-causing SIK1 sequence variations were associated with decreased expression of $A R C$ and other synaptic activity response element genes such as NR4A1(Proschel et al., 2017). Our result further confirmed this notion (Fig. 2l). More interestingly, our transcriptome profiling analysis also enriched some wellknown epilepsy and autism causing genes in the DEGs dataset, such as SCN1A, SCN2A, SCN9A and GABRB3 (Fig. 2D, 2E and 2H). It was recently revealed that sodium channel Nav1.5 controls epithelial-to-mesenchymal transition and invasiveness in breast cancer cells through its regulation by the SIK1(Gradek et al., 2019), indicating the dysregulation of sodium channel by mutant SIK1 might be responsible for the onset of epilepsy in patients with SIK1 syndrome. The detailed mechanism worth further investigation.

Recently it was found that salt-inducible kinases (SIKs) play criticalroles in metabolic homeostasis(Sakamoto et al., 2018). Roles of metabolism in epilepsy are gaining more interest. (Patel, 2018). Metabolic alterations are commonly seen in various forms of epilepsy syndromes, however, the causal relation

Page 5/11 
between metabolic dysfunction and epilepsy is largely unknown (Patel, 2018). In this study, we found epilepsy-causing variant of SIK1 can lead to significant disruption of metabolic homeostasis as demonstrated by the PCA (Fig. 3A), OPLS-DA (Fig. 3B) and vast number of DEGs (Fig. 3D) in the metabolome profiling of cells stably-expressing WT and MUT SIK1. Specifically, cells expressing mutant SIK1 express significantly higher level of Chondroitin 6'-sulfate (Table 2) and lower level of Adenosine compared to cells expressing WT SIK1 (Table 3). Chondroitin sulfate proteoglycans (CSPGs) are predominant components of the extracellular matrix in the central nervous system (CNS). Mice overexpressing chondroitin 6-sulfated chains are more susceptible to seizures(Yutsudo \& Kitagawa, 2015). Adenosine is a well-characterized endogenous anticonvulsant and seizure terminator of the brain. Through a combination of adenosine receptor-dependent and -independent mechanisms, adenosine affects seizure generation, as well as the development of epilepsy and its progression(Weltha, Reemmer, \& Boison, 2019). How does SIK1 contribute to metabolic control of Chondroitin 6'-sulfate and Adenosine also worth further study.

In conclusion, in the present study, we described the seventh case of SIK1 syndrome showing a distinct clinical phenotype compared to previously reported cases, which further expanded the spectrum of SIK1 syndrome. Transcriptome profiling indicated the dysregulation of sodium channel by mutant SIK1 underlie the pathogenesis of SIK1 syndrome. Metabolome profiling support a potent role of Chondroitin 6'-sulfate and Adenosine in initiating epilepsy. Although more mechanistic details are needed, our study enriched our understanding of SIK1 syndrome and epilepsy in general significantly.

\section{Declarations}

\section{Authors' contributions}

Wangshu Xu: project guidance, article writing, Wenqun Zhang: clinical data collection, Lili Cui and Bin Zhu: data statistical analysis, figures plot and charts organization, Tianjie Iv and Wenping Ma: conception and design of all manuscripts.

\section{Funding}

The study was supported by funding from the following: Beijing Municipal Administration of Hospitals [ZYLX201827], the National Natural Science Foundation of China [81803909]

\section{Data availability}

The data that support the findings of this study are available from the corresponding authors upon reasonable request.

\section{Ethical Publication Statement}

We confirm that we have read the Journal's position on issues involved in ethical publication and affirm that this report is consistent with those guidelines. The ethics committee of Beijing Tiantan Hospital of Capital Medical University approved the study. Blood samples collection were performed in accordance with the Declaration of Helsinki.

\section{Disclosure}

None of the authors has any conflict of interest to disclose.

\section{References}

1. Al-Hakim, A. K., Goransson, O., Deak, M., Toth, R., Campbell, D. G., Morrice, N. A., . . Alessi, D. R. (2005). 14-3-3 cooperates with LKB1 to regulate the activity and localization of QSK and SIK. J Cell Sci, 118(Pt 23), 5661-5673. doi:10.1242/jcs.02670

2. Bertorello, A. M., \& Zhu, J. K. (2009). SIK1/SOS2 networks: decoding sodium signals via calcium-responsive protein kinase pathways. Pflugers Arch, 458(3), 613-619. doi:10.1007/s00424-009-0646-2

3. Chen, F., Chen, L., Qin, Q., \& Sun, X. (2019). Salt-Inducible Kinase 2: An Oncogenic Signal Transmitter and Potential Target for Cancer Therapy. Front Oncol, 9, 18. doi:10.3389/fonc. 2019.00018

4. Chen, H., Vanhoutte, P. M., \& Leung, S. W. S. (2019). Acute activation of endothelial AMPK surprisingly inhibits endothelium-dependent hyperpolarizationlike relaxations in rat mesenteric arteries. Br J Pharmacol, 176(16), 2905-2921. doi:10.1111/bph.14716

5. Du, W. Q., Zheng, J. N., \& Pei, D. S. (2016). The diverse oncogenic and tumor suppressor roles of salt-inducible kinase (SIK) in cancer. Expert Opin Ther Targets, 20(4), 477-485. doi:10.1517/14728222.2016.1101452

6. Feldman, J. D., Vician, L., Crispino, M., Hoe, W., Baudry, M., \& Herschman, H. R. (2000). The salt-inducible kinase, SIK, is induced by depolarization in brain. J Neurochem, 74(6), 2227-2238. doi:10.1046/j.1471-4159.2000.0742227.x

7. Gradek, F., Lopez-Charcas, O., Chadet, S., Poisson, L., Ouldamer, L., Goupille, C., . . Roger, S. (2019). Sodium Channel Nav1.5 Controls Epithelial-toMesenchymal Transition and Invasiveness in Breast Cancer Cells Through its Regulation by the Salt-Inducible Kinase-1. Sci Rep, 9(1), 18652. doi:10.1038/s41598-019-55197-5

8. Hansen, J., Snow, C., Tuttle, E., Ghoneim, D. H., Yang, C. S., Spencer, A., . . Paciorkowski, A. R. (2015). De novo mutations in SIK1 cause a spectrum of developmental epilepsies. Am J Hum Genet, 96(4), 682-690. doi:10.1016/j.ajhg.2015.02.013

9. Horike, N., Takemori, H., Katoh, Y., Doi, J., Min, L., Asano, T., . . Okamoto, M. (2003). Adipose-specific expression, phosphorylation of Ser794 in insulin receptor substrate-1, and activation in diabetic animals of salt-inducible kinase-2. J Biol Chem, 278(20), 18440-18447. doi:10.1074/jbc.M211770200 
10. Jaleel, M., Villa, F., Deak, M., Toth, R., Prescott, A. R., Van Aalten, D. M., \& Alessi, D. R. (2006). The ubiquitin-associated domain of AMPK-related kinases regulates conformation and LKB1-mediated phosphorylation and activation. Biochem J, 394(Pt 3), 545-555. doi:10.1042/BJ20051844

11. Kao, S. H., Wang, W. L., Chen, C. Y., Chang, Y. L., Wu, Y. Y., Wang, Y. T., . . Yang, P. C. (2015). Analysis of Protein Stability by the Cycloheximide Chase Assay. Bio Protoc, 5(1). doi:10.21769/BioProtoc.1374

12. Li, M. M., Datto, M., Duncavage, E. J., Kulkarni, S., Lindeman, N. I., Roy, S., . . Nikiforova, M. N. (2017). Standards and Guidelines for the Interpretation and Reporting of Sequence Variants in Cancer: A Joint Consensus Recommendation of the Association for Molecular Pathology, American Society of Clinical Oncology, and College of American Pathologists. J Mol Diagn, 19(1), 4-23. doi:10.1016/j.jmoldx.2016.10.002

13. Marini, C., Mei, D., Temudo, T., Ferrari, A. R., Buti, D., Dravet, C., . . Guerrini, R. (2007). Idiopathic epilepsies with seizures precipitated by fever and SCN1A abnormalities. Epilepsia, 48(9), 1678-1685. doi:10.1111/j.1528-1167.2007.01122.x

14. Papandreou, A., McTague, A., Trump, N., Ambegaonkar, G., Ngoh, A., Meyer, E., . . Kurian, M. A. (2016). GABRB3 mutations: a new and emerging cause of early infantile epileptic encephalopathy. Dev Med Child Neurol, 58(4), 416-420. doi:10.1111/dmcn.12976

15. Patel, M. (2018). A Metabolic Paradigm for Epilepsy. Epilepsy Curr, 18(5), 318-322. doi:10.5698/1535-7597.18.5.318

16. Proschel, C., Hansen, J. N., Ali, A., Tuttle, E., Lacagnina, M., Buscaglia, G., . . Paciorkowski, A. R. (2017). Epilepsy-causing sequence variations in SIK1 disrupt synaptic activity response gene expression and affect neuronal morphology. Eur J Hum Genet, 25(2), 216-221. doi:10.1038/ejhg.2016.145

17. Reynolds, C., King, M. D., \& Gorman, K. M. (2020). The phenotypic spectrum of SCN2A-related epilepsy. Eur J Paediatr Neurol, $24,117-122$. doi:10.1016/j.ejpn.2019.12.016

18. Sakamoto, K., Bultot, L., \& Goransson, O. (2018). The Salt-Inducible Kinases: Emerging Metabolic Regulators. Trends Endocrinol Metab, 29(12), 827-840. doi:10.1016/j.tem.2018.09.007

19. Sun, Z., Jiang, Q., Li, J., \& Guo, J. (2020). The potent roles of salt-inducible kinases (SIKs) in metabolic homeostasis and tumorigenesis. Signal Transduct Target Ther, 5(1), 150. doi:10.1038/s41392-020-00265-w

20. Taub, M., Springate, J. E., \& Cutuli, F. (2010). Targeting of renal proximal tubule Na,K-ATPase by salt-inducible kinase. Biochem Biophys Res Commun, 393(3), 339-344. doi:10.1016/j.bbrc.2010.02.037

21. Wang, J., Christison, T. T., Misuno, K., Lopez, L., Huhmer, A. F., Huang, Y., \& Hu, S. (2014). Metabolomic profiling of anionic metabolites in head and neck cancer cells by capillary ion chromatography with Orbitrap mass spectrometry. Anal Chem, 86(10), 5116-5124. doi:10.1021/ac500951v

22. Wein, M. N., Foretz, M., Fisher, D. E., Xavier, R. J., \& Kronenberg, H. M. (2018). Salt-Inducible Kinases: Physiology, Regulation by cAMP, and Therapeutic Potential. Trends Endocrinol Metab, 29(10), 723-735. doi:10.1016/j.tem.2018.08.004

23. Weltha, L., Reemmer, J., \& Boison, D. (2019). The role of adenosine in epilepsy. Brain Res Bull, 151, 46-54. doi:10.1016/j.brainresbull.2018.11.008

24. Wiklund, S., Johansson, E., Sjostrom, L., Mellerowicz, E. J., Edlund, U., Shockcor, J. P., . . Trygg, J. (2008). Visualization of GC/TOF-MS-based metabolomics data for identification of biochemically interesting compounds using OPLS class models. Anal Chem, 80(1), 115-122. doi:10.1021/ac0713510

25. Xiao, J. F., Zhou, B., \& Ressom, H. W. (2012). Metabolite identification and quantitation in LC-MS/MS-based metabolomics. Trends Analyt Chem, 32, 1-14. doi:10.1016/j.trac.2011.08.009

26. Yutsudo, N., \& Kitagawa, H. (2015). Involvement of chondroitin 6-sulfation in temporal lobe epilepsy. Exp Neurol, 274(Pt B), 126-133. doi:10.1016/j.expneurol.2015.07.009

\section{Tables}

Table 1 The prediction of the identified variant in SIK1

\begin{tabular}{|lllllll|}
\hline Gene name & Position & Transcript & Substitution & Polyphen2 & SIFT & MutationTaster \\
\hline SIK1 & chr21:44840206 & NM_173354 & c.880G>A (p.A294T) & 0.151 & 0.057 & 0.09 (Tolerated) \\
& & & & (Tolerated) & (Tolerated) & \\
\hline
\end{tabular}

Table 2 Top upregulated metabolites in MUT SIK1-expressing cells 


\begin{tabular}{|c|c|c|c|c|c|c|c|c|}
\hline MS2 name & MS2 score & MS1 name & MS1 ppm & SuperClass & MEAN mut & MEAN wt & P-VALUE & Q-VALL \\
\hline & & $\begin{array}{l}\text { 3-Hydroxy-2- } \\
\text { methylpyridine-4,5- } \\
\text { dicarboxylate }\end{array}$ & -19.65344592 & & 0.08759341 & 0.013345734 & 0.026278367 & 0.0457 \\
\hline & & Geranoyl-CoA & -6.26983204 & & 0.175250632 & 0.046524568 & 7.49623E-05 & 0.0004 \\
\hline & & Limocitrin 3-rutinoside & -20.34597893 & & 0.126643516 & 0.043688416 & $1.91801 \mathrm{E}-06$ & 3.5246: \\
\hline & & trans-Dec-2-enoyl-CoA & -7.197870068 & & 0.255501641 & 0.091528874 & 0.000347944 & 0.0013 \\
\hline & & Octanoyl-CoA & 17.33389665 & & 0.270798098 & 0.105674301 & 0.000218747 & 0.0009 \\
\hline & & 4-Pyridoxate & -0.726428636 & & 0.013802608 & 0.005648454 & 0.004571927 & 0.0101 \\
\hline & & Chondroitin 6'-sulfate & 2.248718663 & & 0.020487145 & 0.009074711 & 0.032208181 & 0.0540 \\
\hline \multirow{2}{*}{ Octylamine } & & Decanoyl-CoA & -9.965257107 & & 0.260160083 & 0.123261977 & 0.001781746 & 0.0048 \\
\hline & & $\begin{array}{l}2 '-(6- \\
\text { Acetylglucosyl)astragalin }\end{array}$ & -19.03029101 & & 0.193565826 & 0.094479884 & 8.95023E-07 & 2.5844 \\
\hline \multirow{6}{*}{$\begin{array}{l}\text { 2,2,6,6- } \\
\text { Tetramethyl- } \\
\text { 4- } \\
\text { piperidinone }\end{array}$} & 0.7600918 & & & $\begin{array}{l}\text { Organoheterocyclic } \\
\text { compounds }\end{array}$ & 0.240818675 & 0.126519997 & 0.048359193 & 0.0739 \\
\hline & & $\begin{array}{l}\text { 6-(\{3-[2-(3,4- } \\
\text { dihydroxyphenyl)-5- } \\
\text { hydroxy-4-oxo-7-\{[3,4,5- } \\
\text { trihydroxy-6- } \\
\text { (hydroxymethyl)oxan-2- } \\
\text { yl]oxy\}-3,4-dihydro-2H-1- } \\
\text { benzopyran-8-yl]-5- } \\
\text { hydroxy-2-(4- } \\
\text { hydroxyphenyl)-4-oxo- } \\
\text { 3,4-dihydro-2H-1- } \\
\text { benzopyran-7- } \\
\text { yl\}oxy)-3,4,5- } \\
\text { trihydroxyoxane-2- } \\
\text { carboxylic acid }\end{array}$ & 4.9969969 & & 0.158673004 & 0.087740392 & 0.003001404 & 0.0072 \\
\hline & & Disialyllactose & -15.14051583 & & 0.080879617 & 0.048284228 & 0.017159762 & 0.0321 \\
\hline & & $\begin{array}{l}\text { 6-\{[3-(\{6- } \\
\text { [(acetyloxy)methyl]-3,4,5- } \\
\text { trihydroxyoxan-2- } \\
\text { yl\}oxy)-5-hydroxy-4-oxo- } \\
\text { 2-(3,4,5- } \\
\text { trihydroxyphenyl)-4H- } \\
\text { chromen-7-yl]oxy\}-3,4,5- } \\
\text { trihydroxyoxane-2- } \\
\text { carboxylic acid }\end{array}$ & 19.69013442 & & 0.026152406 & 0.016057098 & 0.002791682 & 0.0068 \\
\hline & & Cobalt-precorrin 4 & -19.36032328 & & 0.069139934 & 0.043644284 & 0.010955448 & 0.0217 \\
\hline & & 3,3'-Dichlorobenzidine & 13.66528388 & & 0.021062997 & 0.013754462 & 0.03469798 & 0.0573 \\
\hline \multirow[t]{4}{*}{ Nicotine } & 0.9777666 & & & $\begin{array}{l}\text { Organoheterocyclic } \\
\text { compounds }\end{array}$ & 0.087092458 & 0.05835246 & 0.041418195 & 0.0658 \\
\hline & & $\begin{array}{l}\text { trans-Zeatin riboside } \\
\text { diphosphate }\end{array}$ & -23.73059867 & & 0.169012022 & 0.114509646 & 0.000102357 & 0.0005 \\
\hline & & 4'-Demalonylsalvianin & 19.46906933 & & 0.129921706 & 0.097713766 & 0.024217792 & 0.0428 \\
\hline & & $\begin{array}{l}\text { Kaempferol 3-(6'- } \\
\text { acetylgalactoside) 7- } \\
\text { rhamnoside }\end{array}$ & -10.14565272 & & 0.274728893 & 0.218539381 & 0.003959736 & 0.0089 \\
\hline $\begin{array}{l}\text { 1-(8-[3]- } \\
\text { ladderane- } \\
\text { octanoyl-2- } \\
\text { (8-[3]- } \\
\text { ladderane- } \\
\text { octanyl)-sn- } \\
\text { glycerol }\end{array}$ & 0.3496904 & & & & 0.16340221 & 0.133473385 & 0.011913424 & 0.0233 \\
\hline
\end{tabular}

Due to technical limitations, table 3 is only available as a download in the Supplemental Files section. 
Figures

A.

$\begin{array}{lll}\text { Father } & \text { Mother } & \text { Patient }\end{array}$

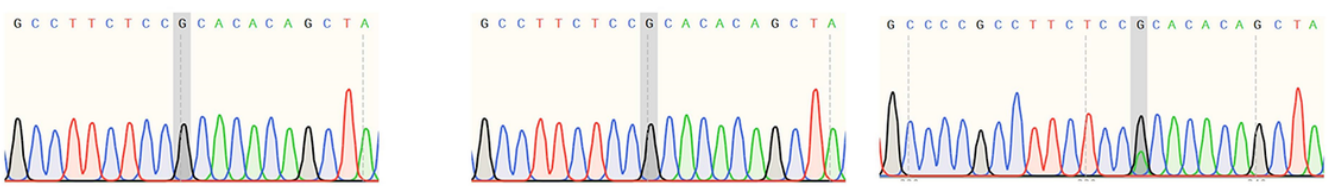

B.

Species/Abbrv

1. sp $Q 60670 \mid$ SIK1 MOUSE

2. sp P57059 SIK1 HUMAN

3. sp Q9R1U5 SIK1 RAT

4. $\operatorname{tr}$ H2QL37 SIK1 Chimpanzee

5. $\operatorname{tr}$ A0A1D5PL73 SIK1 CHICKEN

6. $\operatorname{tr}$ A0A287ALS4 SIK1 PIG

7. $\operatorname{tr}$ E1BNS8 SIK1 BOVIN

8. $\operatorname{tr} \mid$ A0A1U7QMJ7 $\mid$ SIK1 hamster

10. $\operatorname{tr}|\mathrm{Q} 502 \mathrm{~F} 3| \mathrm{SIK} 1$ Zebrafish

KRITIAQIRQHRWMQADPTLLQQDDPAFD

KRITIAQIRQHRWMQADPTLLQQDDPAFS VGYTSNLGDYNEQVLGIMQAL

RRITIAQIRQHRWMRAEPCLPGPACPAFSAHSYTSNLGDYDEQALG IMQTL

RITISQIKQHKWMQADPSLRQQQSLSFSMQNYNSNLGDYNEQVLGIMQTLG

KRISMAQIRQHRWMQADPSLLPPACPAFS LSYNSSLGDYDEQALAIMQAL

KRITIAQIRQHRWMQAEPALVLPACPGFS L LGYTSSVGDYDEQALGIMQML

RIT IAQIRQHRWMRAEPCFPGPACPTFS HSYSSNLGDYDEQALGIMQTL

C.

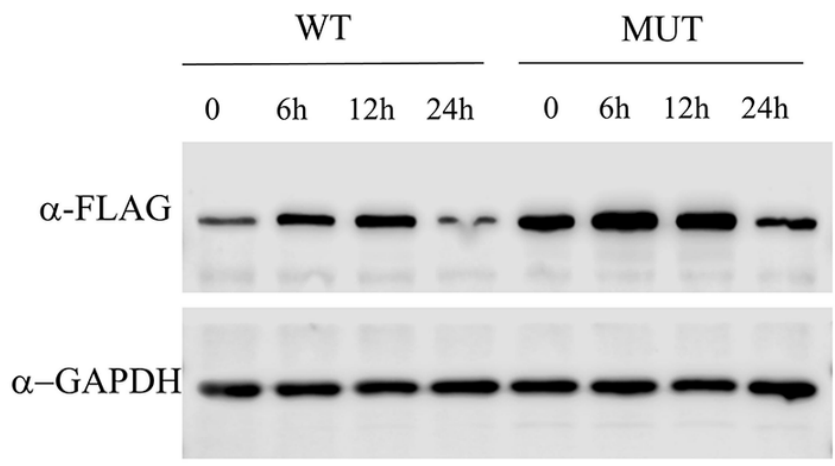

Figure 1

Novel de novo heterozygous mutation in SIK1. A. Whole exome sequencing identified a c.880G >A variation resulting in a p.A294T amino acid substitution, confirmed de novo by Sanger sequencing of proband and parents. B. Aligned and compared amino acid sequences of SIK1 among species. C. Protein stability test of mutant SIK1. Cycloheximide Chase Assay for A297T mutant SIK1 and WT control. Representative images from 2-3 independent experiments. 
A.

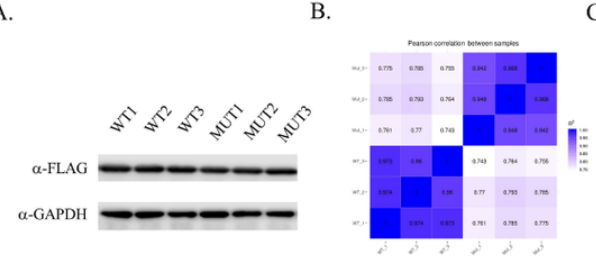

D.

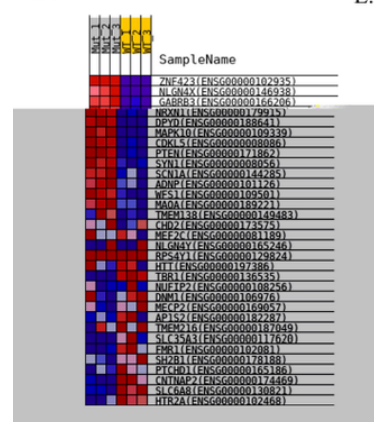

G.

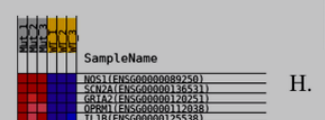

E.

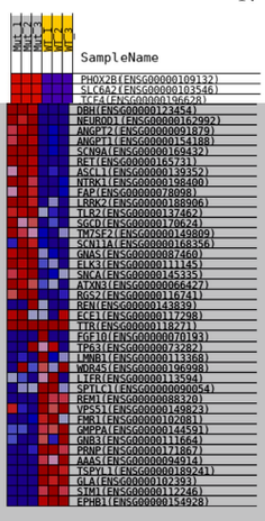

F.
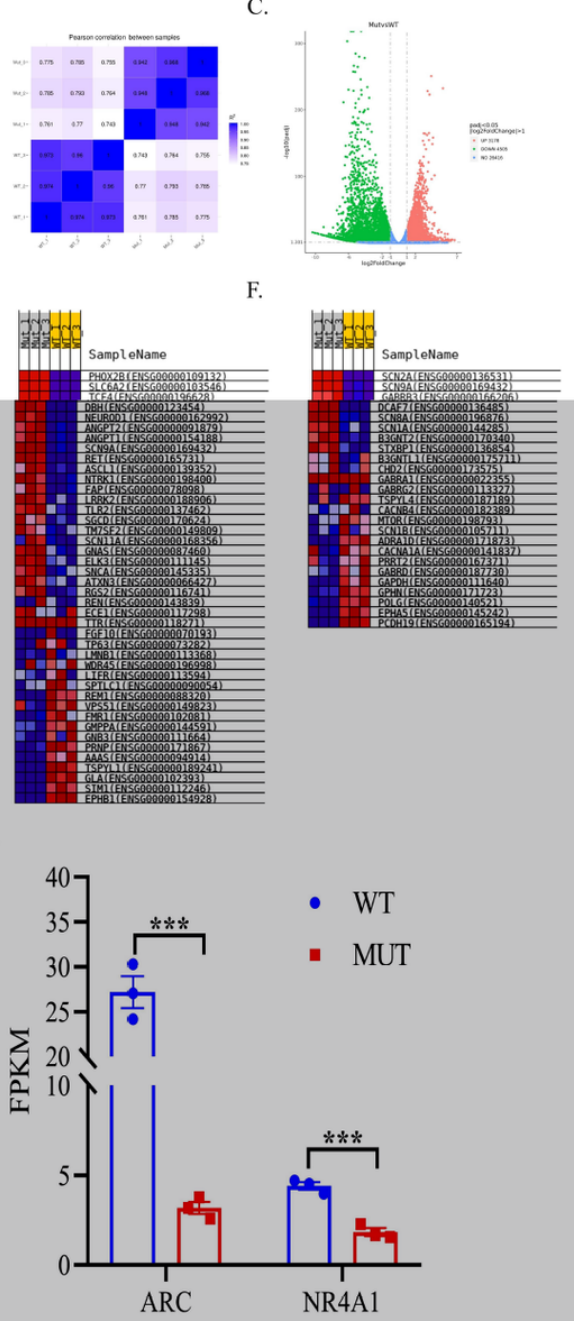

Figure 2

Transcriptomic dysregulation confirmed the pathogenicity of novel SIK1 mutation. A. Western blot confirmed the successful expression of transfected plasmids. B. Pearson correlation between samples showing dissimilarity between groups. C. Volcano plot indicating transcriptomic changes between groups. Significantly upregulated (FC $>1$, FDR-adj. $p$ value $<0.05$, red) and downregulated (FC<-1, FDR-adj. p value $<0.05$, green) genes are shown. D. Heatmap showing significantly differentially expressed genes enriched in "INFANTILE_SEVERE_MYOCLONIC_EPILEPSY" using DisGeNET enrichment method. E. Heatmap showing significantly differentially expressed genes enriched in "AUTONOMIC_NERVOUS_SYSTEM_DISORDERS" using DisGeNET enrichment method. F. Heatmap showing significantly differentially expressed genes enriched in "AUTISTIC_BEHAVIOR" using DisGeNET enrichment method. G. Heatmap showing significantly differentially expressed genes enriched in "TEMPORAL_LOBE_EPILEPSY" using Disease Ontology enrichment method. H. Comparison of indicated genes between WT and Mutant groups by RT-qPCR. Unpaired two-sided Student' s t-test, data are represented as mean $\nabla$ SD. I. Comparison of indicated genes between WT and Mutant groups by RNA-seq (left) and RT-qPCR (right). Unpaired two-sided Student' s t-test, data are represented as mean $\mathrm{I}$ SD. 
A.

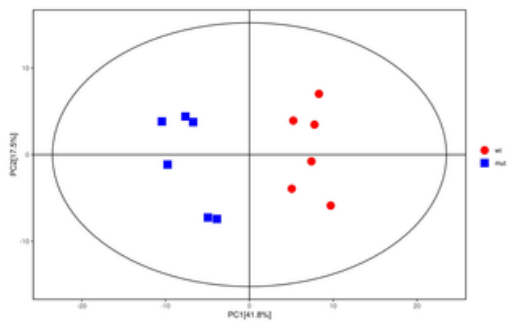

D.

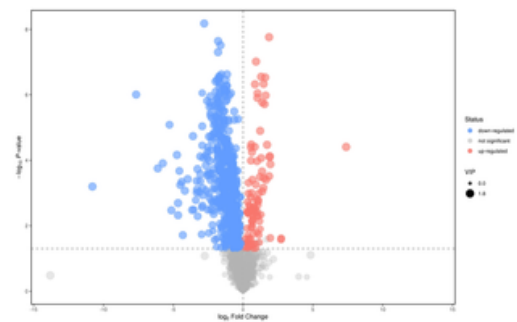

B.

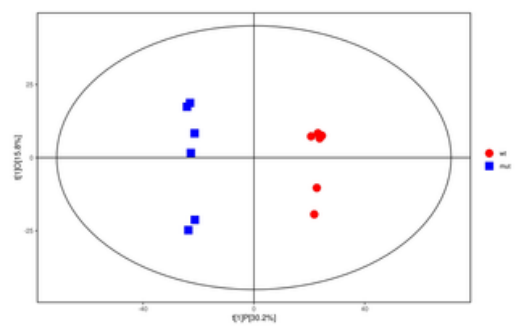

E.

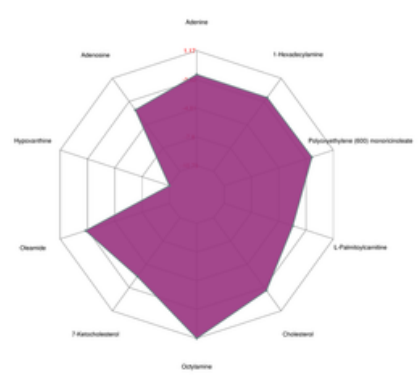

C.

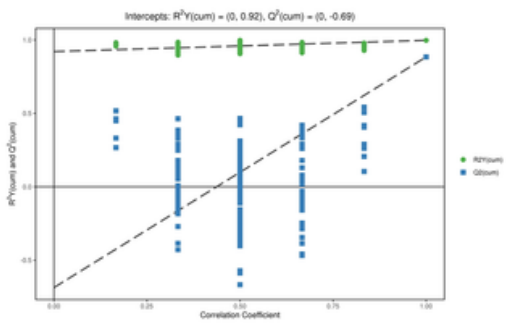

F.

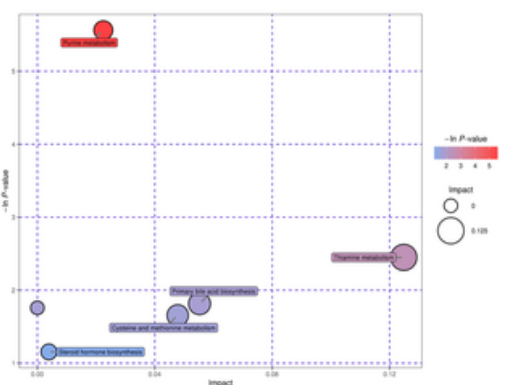

Figure 3

Metabolic dysregulation confirmed the pathogenicity of novel SIK1 mutation. A. Principal component analysis of metabolic pattern between WT group and MUT group. B. Orthogonal projections to latent structures-discriminant analysis of metabolic pattern between WT group and MUT group. C. Permutation test of OPLS-DA model analysis of metabolic pattern between WT group and MUT group. D. Volcano plot indicating metabolic changes between groups. Significantly upregulated (FC $>1$, FDR-adj. p value $<0.05$, red) and downregulated (FC<-1, FDR-adj. p value $<0.05$, blue) genes are shown. E. Radar chart showing general quantification of differentially expressed metabolites between groups. F. Pathway enrichment analysis of differentially expressed metabolites between groups.

\section{Supplementary Files}

This is a list of supplementary files associated with this preprint. Click to download.

- Table3.docx 